\title{
Superconducting nanowire single-photon sensing of cerebral blood flow
}

\author{
Nisan Ozana $\odot,{ }^{a}, \uparrow * *$ Alexander I. Zavriyev, ${ }^{\text {a, }}{ }^{\dagger}$ Dibbyan Mazumder, ${ }^{\text {a }}$ \\ Mitchell Robinson $\odot,{ }^{\text {a,b }}$ Kutlu Kaya, ${ }^{\text {a }}$ Megan Blackwell, ${ }^{\mathrm{c}}$ \\ Stefan A. Carp $\odot,{ }^{a}$ and Maria Angela Franceschini ${ }^{a}$ \\ ${ }^{a}$ Massachusetts General Hospital, Harvard Medical School, \\ Optics at Athinoula A. Martinos Center for Biomedical Imaging, \\ Department of Radiology, Boston, Massachusetts, United States \\ ${ }^{b}$ Massachusetts Institute of Technology, Health Sciences and Technology Program, \\ Cambridge, Massachusetts, United States \\ ${ }^{c}$ Massachusetts Institute of Technology Lincoln Laboratory, Lexington, \\ Massachusetts, United States
}

\begin{abstract}
Significance: The ability of diffuse correlation spectroscopy (DCS) to measure cerebral blood flow (CBF) in humans is hindered by the low signal-to-noise ratio (SNR) of the method. This limits the high acquisition rates needed to resolve dynamic flow changes and to optimally filter out large pulsatile oscillations and prevents the use of large source-detector separations $(\geq 3 \mathrm{~cm})$, which are needed to achieve adequate brain sensitivity in most adult subjects.

Aim: To substantially improve SNR, we have built a DCS device that operates at $1064 \mathrm{~nm}$ and uses superconducting nanowire single-photon detectors (SNSPD).

Approach: We compared the performances of the SNSPD-DCS in humans with respect to a typical DCS system operating at $850 \mathrm{~nm}$ and using silicon single-photon avalanche diode detectors.

Results: At a 25-mm separation, we detected $13 \pm 6$ times more photons and achieved an SNR gain of $16 \pm 8$ on the forehead of 11 subjects using the SNSPD-DCS as compared to typical DCS. At this separation, the SNSPD-DCS is able to detect a clean pulsatile flow signal at $20 \mathrm{~Hz}$ in all subjects. With the SNSPD-DCS, we also performed measurements at $35 \mathrm{~mm}$, showing a lower scalp sensitivity of $31 \pm 6 \%$ with respect to the $48 \pm 8 \%$ scalp sensitivity at $25 \mathrm{~mm}$ for both the 850 and $1064 \mathrm{~nm}$ systems. Furthermore, we demonstrated blood flow responses to breath holding and hyperventilation tasks.

Conclusions: While current commercial SNSPDs are expensive, bulky, and loud, they may allow for more robust measures of non-invasive cerebral perfusion in an intensive care setting.

(C) The Authors. Published by SPIE under a Creative Commons Attribution 4.0 Unported License. Distribution or reproduction of this work in whole or in part requires full attribution of the original publication, including its DOI. [DOI: 10.1117/1.NPh.8.3.035006]
\end{abstract}

Keywords: superconducting nanowire detectors; diffuse correlation spectroscopy; cerebral blood flow; pulsatile blood flow.

Paper 21016R received Apr. 13, 2021; accepted for publication Jul. 26, 2021; published online Aug. 19, 2021.

\section{Introduction}

Diffuse correlation spectroscopy (DCS) is a non-invasive optical method for the measurement of blood flow (BF). ${ }^{1}$ In DCS, the tissue is illuminated by a long coherence length near-infrared laser, and the speckle pattern formed by moving scatterers, mostly red blood cells, modulates

*Address all correspondence to Nisan Ozana,nozana@mgh.harvard.edu,nisaneti@gmail.com

${ }^{\dagger}$ These authors contributed equally to the paper. 
the detected light. The decay of the measured temporal intensity autocorrelation function $\left[g_{2}(\tau)\right]$ originated by the speckle fluctuations provides an index of blood flow $\left(\mathrm{BF}_{\mathrm{i}}\right),{ }^{2}$ with units $\mathrm{cm}^{2} / \mathrm{s}$. To maximize the contrast of the measured speckle, single-mode fibers are used, greatly limiting potential photon throughput. Current DCS devices employing single-photon avalanche photodiodes (SPAD) detectors and laser sources at 700 to $850 \mathrm{~nm}$ typically operate at a source-detector (SD) separation of $25 \mathrm{~mm}$ and an acquisition rate of $1 \mathrm{~Hz} .{ }^{3}$ Larger SD separations are desirable for improving brain sensitivity and reducing scalp signal contamination, especially in the adult population. Faster acquisition rates are needed to detect fast BF dynamics and effectively remove the large pulsatile systemic component from the cerebral signals. Unfortunately, the low signalto-noise ratio (SNR) of current devices limits the acquisition rates and prevents the use of SD separations $>2.5 \mathrm{~cm}$.

To improve upon the single speckle limitations of conventional DCS measurements, multiple colocalized detectors are used to increase $\mathrm{SNR}^{4,5}$ and as a proof of principle, at a short separation $(11 \mathrm{~mm})$, Sie et al. ${ }^{6}$ have recently reported a $g_{2}(4 \mu \mathrm{s})$ SNR gain of $32(\sqrt{1024})$ using a $32 \times$ 32 pixel SPAD camera to enable multi-speckle detection. As an alternative, DCS measurements with heterodyne detection have been proposed to improve the SNR through amplification of the signal via a reference arm. Using a fiber Mach-Zehnder interferometer and conventional silicon SPAD detectors, we have shown an increase in the SNR of the autocorrelation curve by a factor of $\sim 2$ and a reduction of $80 \%$ in the coefficient of variation of the fitted $\mathrm{BF}_{\mathrm{i}}$ at long sourcedetector separations $(>30 \mathrm{~mm}) .{ }^{7}$ Further, by increasing the magnitude of the intensity fluctuations, conventional camera sensors can be used, enabling a greater increase in SNR mediated by multi-speckle detection. ${ }^{8,9}$

In addition to acting at the detection side, we have recently proposed to use wavelengths above $1 \mu \mathrm{m}$ to increase DCS SNR. ${ }^{10}$ Larger photon availability and slower autocorrelation function decay contribute to a substantial increase in SNR when using wavelengths around 1050 to $1100 \mathrm{~nm}$ with respect to the wavelengths traditionally used for near-infrared spectroscopy. The problem of operating at these longer wavelengths is that silicon (Si) SPADs and Si cameras have very low photon efficiencies, indium gallium arsenide (InGaAs) SPADs have a strong afterpulsing probability right where the $g_{2}$ starts to decay (1 to $10 \mu \mathrm{s}$ ), and InGaAs cameras are not fast enough to detect the initial autocorrelation decay.

Here, we propose to use superconducting nanowire single-photon detectors (SNSPDs) to operate DCS at $1064 \mathrm{~nm}$ and overcome other detectors limitations.

SNSPDs were demonstrated 20 years ago, ${ }^{11}$ following an observation that the superconductivity of a lead film can be disrupted by a laser beam. ${ }^{12}$ SNSPDs operate below the boiling point of liquid helium, $<4.2 \mathrm{~K}$, and consist of a thin film of superconducting material patterned in a compact geometry to create a large pixel with high detection efficiency and a high single-photon sensitivity due to the nanoscale cross-section. When a photon reaches the nanowire, superconductivity is locally broken and the impedance is increased, creating a voltage pulse. After the photon is absorbed, superconductivity quickly recovers and the SNSPD is ready to detect the next photon.

SNSPDs have several advantages over SPADs, such as recovery time $(<50 \mathrm{~ns})$, timing precision $(<80 \mathrm{ps})$, photon efficiency $(>80 \%)$, and broad wavelength sensitivity (600 to $1550 \mathrm{~nm}) .{ }^{13}$ SNSPDs are also superior to InGaAs SPADs with respect to dark count rate ( $\sim$ CPS versus $<10$ KCPS) ${ }^{14}$ and, more importantly, do not have afterpulsing issues. In the following Table 1, we report the key metrics of the detectors used here [single-photon avalanche diode detectors (Si-SPAD) SPCM-NIR-14-FC, Excelitas and SNSPD Opus One, Quantum Opus] and the one of a commercially available InGaAs SPAD (PDM-IR, micro photon detectors).

SNSPDs are used in optical quantum information, telecommunication, and space communication. ${ }^{15}$ SNSPDs have also recently been used in fluorescence lifetime imaging microscopy ${ }^{16}$ but never before in biomedical applications. To our knowledge, this is the first application of SNSPDs in humans to improve DCS performance.

In this work, we propose a new DCS system that includes a laser emitting at $1064 \mathrm{~nm}$ and two SNSPD detectors. To demonstrate the advantages of the SNSPD-DCS system at $1064 \mathrm{~nm}$ with respect to standard DCS, we performed simultaneous measurements on the forehead of 11 subjects with the SNSPD-DCS and an Si-SPAD-based DCS system operating at $850 \mathrm{~nm}$. 
Table 1 Typical key specifications of three single-photon counting detectors used for DCS.

\begin{tabular}{|c|c|c|c|c|c|c|}
\hline & $\begin{array}{l}\text { Dead } \\
\text { time }\end{array}$ & $\begin{array}{c}\text { Dark } \\
\text { count rate }\end{array}$ & $\begin{array}{l}\text { Timing } \\
\text { resolution } \\
\text { (ps) }\end{array}$ & $\begin{array}{c}\text { PDE } \\
850 \mathrm{~nm} \\
(\%)\end{array}$ & $\begin{array}{c}\text { PDE } \\
1064 \mathrm{~nm} \\
(\%)\end{array}$ & $\begin{array}{l}\text { Operating } \\
\text { temperature }\end{array}$ \\
\hline Si-SPAD (excelitas) & $<25$ ns & $<1500$ CPS & 350 & up to 58 & $<\mathbf{3}$ & 278 to $343 \mathrm{~K}$ \\
\hline InGaAs SPAD (MPD) & $>10 \mu \mathrm{s}^{\mathrm{a}}$ & $<10,000$ CPS & $<130$ & $<5$ & $\sim 32$ & 228 to $243 \mathrm{~K}$ \\
\hline SNSPD (quantum Opus) & $<50$ ns & $1 \mathrm{CPS}$ & $<80$ & $>80$ & $>80$ & 2 to $3.1 \mathrm{~K}$ \\
\hline
\end{tabular}

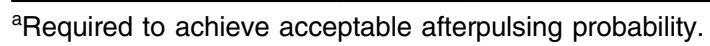

\section{Materials and Methods}

\subsection{Subject Recruitment}

For this study, we enrolled 11 healthy subjects (five males, six females, mean age $29 \pm 9$ years, all right-handed, and non-smokers) between August 2020 and September 2020. The study was reviewed and approved by the Mass General Brigham Human Research Committee (IRB \#2019P003074).

\subsection{SNSPD-DCS System at $1064 \mathrm{~nm}$}

The SNSPD-DCS system consists of a long coherence length laser at $1064 \mathrm{~nm}$ (CL1064-300$\mathrm{SO}$, CrystaLaser). At this wavelength, we can illuminate the skin with $100 \mathrm{~mW}$ of power. In fact, following the American National Standards Institute for Safe Use of Lasers ANSI Z136.1 2007 Tables 7, 8a, and 8b, pages 77-79, the maximum permitted radiant exposure for continuous skin illumination at $1064 \mathrm{~nm}$ is $1 \mathrm{~W} / \mathrm{cm}^{2}$, and for an illumination spot larger than a 1-mm diameter, a 3.5-mm spot size can be considered, which leads to $100 \mathrm{~mW}$ of light power. This power is more than double the allowable power at $850 \mathrm{~nm}(38 \mathrm{~mW}$ when estimated for the same illumination geometry). In the study, light was delivered to the tissue via a $200-\mu \mathrm{m}$ multimode fiber terminated with a $3.5-\mathrm{mm}$ prism to direct the light perpendicular to the fiber. A holographic diffuser located between the fiber and the prism expanded the beam spot at the skin to about 3-mm OD diameter. The backscattered photons were collected at 5,25 , and $35 \mathrm{~mm}$ separations from the source by single-mode fibers $(5-\mu \mathrm{m}$ core diameter, $780 \mathrm{HP}$, Thorlabs) terminated with $1-\mathrm{mm}$ prisms (Fig. 1). The 25- and 35-mm separation fibers were connected to two SNSPDs (Quantum Opus, Opus One) operating at $3.1^{\circ} \mathrm{K}$ and with direct current of $7 \mu \mathrm{A}$. These SNSPDs include a cryostat, which needs to be on for about $3 \mathrm{~h}$ to reach the operating low temperature. After that point, the cryostat can be turned off for up to $20 \mathrm{~min}$ at a time whenever the device needs to be moved without the need to restart the cooling procedure. The two SNSPD detectors were optimized for 1064-nm illumination by the manufacturer by tuning the detector's cavity at this wavelength, which led to a photon efficiency of about $88 \%$. For the 5-mm SD separation, we used an Si-SPAD that was part of the conventional DCS system described below since at that distance we had more than enough photons to overcome the very low photon efficiency $(<3 \%)$ of the Si-SPAD at $1064 \mathrm{~nm}$. The arrival times of the photons were collected by a custom FPGAbased multichannel time-tagger, transmitted to a laptop for real-time display at $1 \mathrm{~Hz}$, and later processed for faster acquisition rates.

\subsection{Conventional Si-SPAD Based DCS at $850 \mathrm{~nm}$}

A conventional DCS system operating at $850 \mathrm{~nm}$ was used to compare performances with the SNSPD-DCS system. This custom DCS device included a long coherence length laser (DL852100-SO, CrystaLaser) attenuated to provide $38 \mathrm{~mW}$ at the skin, and four Si-SPAD detectors (SPCM-NIR-14, Excelitas) providing 58\% photon detection efficiency at $850 \mathrm{~nm}$. An optical probe symmetrical to the one used for the SNSPD-DCS hosted a multimode fiber for the source and single-mode fibers for the detectors, one at $5 \mathrm{~mm}$ and two at $25 \mathrm{~mm}$ from the source (Fig. 1). The fourth SPAD was connected to the short separation fiber of the 1064 probe. 


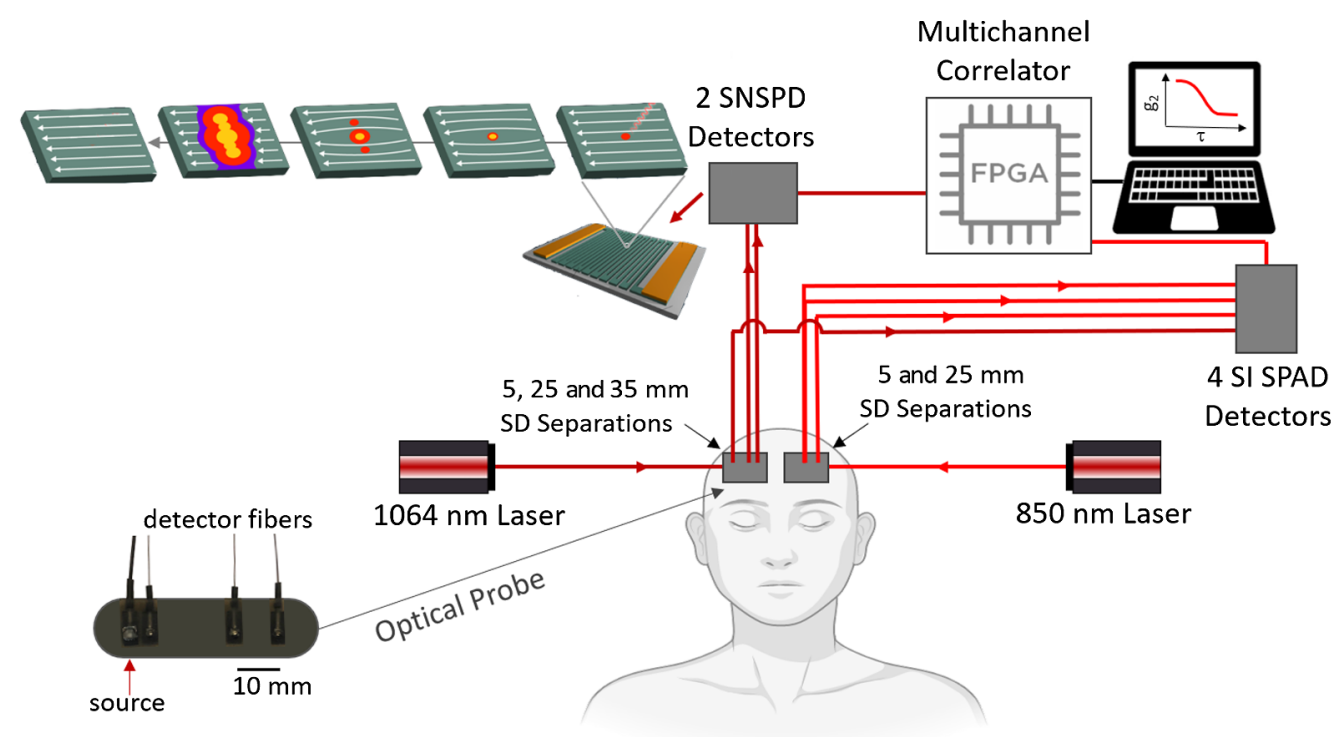

Fig. 1 Schematic diagram of the experimental setup. Two long coherence length lasers, one at $1064 \mathrm{~nm}$ and one at $850 \mathrm{~nm}$, were used to simultaneously illuminate two locations on the forehead of a subject via multimode optical fibers hosted in two symmetrical optical probes (one shown in details in the low left insert). The probes also included single-mode detector fibers at 5, 25, and $35 \mathrm{~mm}$ from the source. For the 850-nm probe we did not use the $35-\mathrm{mm}$ fiber but used two colocalized fibers at $25 \mathrm{~mm}$ connected to 2 Si-SPAD detectors to increase SNR. The 5-mm fiber in the 1064-nm probe was connected to a Si-SPAD detector, whereas the 25 and $35 \mathrm{~mm}$ separation fibers were connected to two SNSPD detectors. The signals from all detectors was sent to a custom-made FPGA correlator board to digitize the photons arrival times and to a computer to process in real time the temporal autocorrelation function of each detectors and save the data. The figure also shows a graphical rendering of the laser light's interaction with a SNSPD detector.

\subsection{Measurement Protocols}

After a phone screening, on the day of the measurement the subject signed the consent form and was further instructed about the experimental procedures. The subject sat in a testing room adjacent to the control room that housed experimental devices. Both optical probes were channeled through an opening between the rooms and secured to the subject's forehead as close as possible to the hairline. A pulse oximeter was used to monitor peripheral oxygen saturation $\left(\mathrm{SpO}_{2}\right)$ and heart rate (HR). The pulse oximeter and the DCS data were synchronized via computer time-stamps. During the tasks, room lights were dimmed, and due to COVID-19 precautions, the study staff was only in the same room as the subject during the tourniquet task. Subjects wore a surgical mask during all procedures. A glass window between the two rooms allowed us to observe the subject and communication was done via an intercom. The entire session lasted $<1 \mathrm{~h}$.

Each subject had one probe on each side of the forehead for the experiments. To account for potential regional differences between the left and right hemispheres, we alternated which side each optical probe was on between subjects. We secured the probes with black tape, which also served to minimize ambient light.

\subsubsection{Pressure modulation task}

To assess the brain sensitivity of the 1064-nm SNSPD-DCS system, we conducted a pressure modulation task using a medical-grade tourniquet. ${ }^{17}$ The tourniquet was positioned between the eyebrow and the sensors and loosely wrapped around the head. The tourniquet was tightened for $60 \mathrm{~s}$ to decrease the blood flow to the scalp by compressing the superficial temporal, the supratrochlear, and the supraorbital arteries. The pressure did not to cause any discomfort to the subjects, and compression was repeated three times, with each trial being preceded and followed by a 1-min baseline. 


\subsubsection{Breathing task}

In this set of measurements, subjects engage in two breathing exercises, breath-holding and hyperventilation. The task consisted of a 1-min baseline, breath-holding for as long the subject was able to but no more than $1 \mathrm{~min}$, a 2-min recovery, 1-min hyperventilation, and a 2-min recovery. For breath-holding, subjects were instructed to begin breathing out $5 \mathrm{~s}$ leading up to the task since breath-holding after expiration leads to a more rapid increase in $\mathrm{CBF}$, allowing for shorter breath-holding observation periods. ${ }^{18}$ For hyperventilation, subjects were instructed to attempt to fully exhale and inhale rapidly to maximize the respiratory exchange ratio. The task was repeated three times. Pulse oximeter changes in $\mathrm{SpO}_{2}$ and HR were used to assess subject's compliance.

\subsection{Data Analysis}

For each subject and each trial, at each distance and each wavelength, we computed the temporal autocorrelation functions $\left[g_{2}(\tau)\right]$ at $1 \mathrm{~Hz}$ to fit for slow blood flow changes, and at 10, 20, and $50 \mathrm{~Hz}$ to extract pulsatile blood flow and to estimate its contrast-to noise ratio (CNR). To calculate $\mathrm{BF}_{\mathrm{i}}$, each $g_{2}$ was fitted to the semi-infinite correlation diffusion equation ${ }^{19}$ using fixed optical properties corresponding to the ones of the brain layer, reported in Table $2 .{ }^{20}$ The pulsatile CNR was estimated as the contrast between the FFT amplitude at the pulsation frequency and the noise floor, and a clear signal was defined using a threshold of CNR $>4$.

For the pressure modulation and breathing task comparisons, we considered relative blood flow $\left(\mathrm{rBF}_{\mathrm{i}}\right)$, obtained in each subject and in each trial by normalizing $\mathrm{BF}_{\mathrm{i}}$ by the mean value calculated between 10 and $50 \mathrm{~s}$ of the baseline period.

We calculated $\mathrm{BF}_{\mathrm{i}} \%$ reductions with pressure as $\left(1-\mathrm{rBF}_{\mathrm{i} \text { pressure }}\right) * 100$. Since the two DCS optical sensors were not colocalized, we considered only trials for which the difference between the left and right forehead's reductions at the short separations was small $(<10 \%$ difference) for a period of at least $20 \mathrm{~s}$ during the compression.

To assess subject compliance with the breathing tasks, we examined the pulse oximetry responses. For the breath-holding task, we divided the trials in two groups based on $\mathrm{SpO}_{2}$ decreases lower or $>5 \%$. For the hyperventilation trials, we excluded subjects where we did not observe an increase in hearth rate of at least 10 beats per minute. Each trial was normalized with respect to a $30 \mathrm{~s}$ baseline before breath-holding and hyperventilation.

In addition to $\mathrm{BF}_{\mathrm{i}}$, average photon counts at both wavelengths and all separations were quantified. Gains at the same SD separations were defined as the ratio between the value at 1064 and the value at $850 \mathrm{~nm}$.

To verify the tabulated difference in scattering between 850 and $1064 \mathrm{~nm}$ matched the experimental results we assumed the same blood flow and a fixed absorption coefficient $\left(\mu_{a}\right)$ at each wavelength. Specifically, we first imposed a constant reduced scattering coefficient $\left(\mu_{s}{ }^{\prime}\right)$ of $9.25 \mathrm{~cm}^{-1}$ to fit the $850 \mathrm{~nm}$ autocorrelation curve and extracted the $850 \mathrm{~nm} \mathrm{BF}$. Then, we fit the $\mathrm{g}_{2}$ curve at $1064 \mathrm{~nm}$ with scattering values ranging from 6.5 to $10.5 \mathrm{~cm}^{-1}$ and found which $\mu_{s}{ }^{\prime}$ provided the minimum difference between $\mathrm{BF}_{\mathrm{i}}$ at $1064 \mathrm{~nm}$ and $\mathrm{BF}_{\mathrm{i}}$ found at $850 \mathrm{~nm}$.

Table 2 Reduced scattering coefficients, absorption coefficients and $\mathrm{BF}_{\mathrm{i}}$ in four tissue layers derived from Refs. 20, 30, and 31 and used in the Monte Carlo simulations. For the SNR evaluation, we used the brain optical properties.

\begin{tabular}{|c|c|c|c|c|c|}
\hline \multirow{2}{*}{$\begin{array}{l}\text { Optical properties } \\
\text { tissue layer }\end{array}$} & \multicolumn{2}{|c|}{$\mu_{s}^{\prime}\left(\mathrm{cm}^{-1}\right)$} & \multicolumn{2}{|c|}{$\mu_{a}\left(\mathrm{~cm}^{-1}\right)$} & \multirow[b]{2}{*}{$\mathrm{BF}_{\mathrm{i}}\left(\mathrm{cm}^{2} / \mathrm{s}\right)$} \\
\hline & $1064 \mathrm{~nm}$ & $850 \mathrm{~nm}$ & $1064 \mathrm{~nm}$ & $850 \mathrm{~nm}$ & \\
\hline Scalp & 8.38 & 9.25 & 0.11 & 0.091 & $1 \times 10^{-8}$ \\
\hline Skull & 8.38 & 9.25 & 0.13 & 0.11 & 0 \\
\hline CSF & 0.09 & 0.10 & 0.12 & 0.043 & 0 \\
\hline Brain & 8.38 & 9.25 & 0.17 & 0.20 & $6 \times 10^{-8}$ \\
\hline
\end{tabular}


To evaluate the SNR of the two DCS devices in the most realistic situation, we decided to use human data. For this SNR evaluation, we used the three 1-min baseline periods acquired at the beginning of the pressure modulation tasks. To eliminate physiological noise, we only considered $g_{2}$ values at the diastolic points of the cardiac cycle. The diastolic points were identified from the $10 \mathrm{~Hz} \mathrm{BF}$ time trace at both 850- and 1064-nm short separations, and at $25 \mathrm{~mm}$ at $1064 \mathrm{~nm}$ since in these cases the pulsatile CNR was $>4$. The identified diastolic timepoints were used in all $\mathrm{BF}_{\mathrm{i}}$ traces, including the $25 \mathrm{~mm}$ at $850 \mathrm{~nm}$ and of $35 \mathrm{~mm}$ at $1064 \mathrm{~nm}$ where the higher noise made the diastolic points unidentifiable. The mean $\left[g_{2}(\tau)\right]$ and the standard deviation of $\left[g_{2}(\tau)\right]$ were calculated at a correlation lag time $\tau=4 \mu$ s by averaging 50 dyastolic $g_{2}$ acquired at $10 \mathrm{~Hz}$. Finally, the SNR was calculated using the following expression:

$$
\mathrm{SNR}\left|g_{2}(\tau)\right|=\operatorname{mean}\left[g_{2}(\tau)-1\right] / \operatorname{STD}\left[g_{2}(\tau)\right]
$$

We also compared the experimental SNR with the theoretical SNR as described in the noise model in Refs. 21 and 22. Based on this model, the theoretical standard deviation $[\sigma(\tau)]$ at each time delay $\tau$ is given by

$$
\begin{aligned}
\sigma(\tau)= & \sqrt{\frac{T}{t}}\left[\beta^{2} \frac{\left(1+e^{-2 \Gamma T}\right)\left(1+e^{-2 \Gamma \tau}\right)+2 m\left(1-e^{-2 \Gamma T}\right) e^{-2 \Gamma \tau}}{\left(1-e^{-2 \Gamma T}\right)}+2\langle n\rangle^{-1} \beta\left(1+e^{-2 \Gamma \tau}\right)\right. \\
& \left.+\langle n\rangle^{-2}\left(1+\beta e^{-\Gamma \tau}\right)\right]^{1 / 2}
\end{aligned}
$$

where $t$ is the integration time; $T$ is the bin width; $\Gamma$ is the exponential decay rate of $g_{1}(\tau)$, which depends on $\mathrm{BF}_{\mathrm{i}} ;\langle n\rangle$ is the average number of recorded photons multiplied by the bin width $\mathrm{T}$; $m$ is the is auto-correlation lag bin index; and $\beta$ is the coherence factor. Actual photon counts and average $\beta$ and $\mathrm{BF}_{\mathrm{i}}$ were inputs to the model, and the same brain optical properties used for the experimental calculations were used for the noise model.

Moreover, we evaluated the expected brain sensitivity with Monte Carlo simulations. The MCX software package ${ }^{23}$ was used to simulate photon transport and momentum transfer in a realistic brain geometry. ${ }^{24}$ For this forward model, we used a four-layer MRI-derived volumetric geometry segmented into scalp, skull, cerebrospinal fluid (CSF), gray, and white matter (brain), with $1 \mathrm{~mm} \times 1 \mathrm{~mm} \times 1 \mathrm{~mm}$ spatial resolution. For each of the four tissue types, we used the optical properties and $\mathrm{BF}_{\mathrm{i}}$ reported in Table 2 . The probe consisted of a collinear arrangement of a source and seven detectors at distances $(r)=5,10,15,20,25,30,35$, and $40 \mathrm{~mm}$ from the source and was placed in a location in the head with extracerebral thickness of about $14 \mathrm{~mm}$. Auto-correlation curves obtained from MC simulations were post-processed to add statistical noise using the same noise model mentioned in the previous section,${ }^{21}$ then fit with the same semi-infinite correlation diffusion model as the experimental data.

For the photon counts, we used the experimental values averaged across all subjects: 114,000 counts per second (CPS) at $25 \mathrm{~mm}$ for $1064 \mathrm{~nm}, 11,000 \mathrm{CPS}$ at $25 \mathrm{~mm} 850 \mathrm{~nm}$, and 12,000 CPS at $35 \mathrm{~mm}$ at $1064 \mathrm{~nm}$. To estimate brain sensitivity, we increased the $\mathrm{BF}_{\mathrm{i}}$ in the brain layer by $20 \%$ to $7.2 \times 10^{-9} \mathrm{~cm}^{2} / \mathrm{s}\left(\mathrm{BF}_{\mathrm{i} \text { perturb }}\right)$ while keeping scalp, skull and $\mathrm{CSF} \mathrm{BF}_{\mathrm{i}}$ constant and quantified the increase in apparent $\mathrm{BF}_{\mathrm{i}}$ fit with a semi-infinite model. To estimate $\mathrm{BF}_{\mathrm{i}}$ reduction due to scalp contamination at different SD separations, we simulated a scalp BF reduction of $85 \%$ (as found in our pressure modulation experiments) and quantified the decrease in apparent $\mathrm{BF}_{\mathrm{i}}$ fit with the same semi-infinite model. To determine the brain contrast-to-noise ratio at each SD separation $(r)$ and wavelength $(\lambda)$, we used the perturbation and baseline estimated $\mathrm{BF}_{\mathrm{i}}$ from fitting and estimated the standard deviation of fitted $\mathrm{BF}_{\mathrm{i}}$ over 120 noise realizations assuming a 1-s integration time. The brain CNR was estimated for each experimental SD separation and wavelength as

$$
\operatorname{CNR}(r, \lambda)=\frac{B F_{i \text { perturb }}(r, \lambda)-B F_{i \text { baseline }}(r, \lambda)}{\sigma_{B F i}(r, \lambda)}
$$




\section{Results}

\subsection{SNR Comparison}

As described in Sec. 2.5, to assess the SNR of the SNSPD-DCS with respect to conventional DCS, we considered the baseline periods of the pressure modulation trials and averaged $50 g_{2}$ curves acquired at $10 \mathrm{~Hz}$ at the diastolic point of the arterial pulsation cycle. Figure 2(a) shows the resulting autocorrelation curves at $25 \mathrm{~mm}$ for the $1064 \mathrm{~nm}$ and the $850 \mathrm{~nm}$ DCS systems in a representative subject. As expected, the autocorrelation curve at $1064 \mathrm{~nm}$ has a slower decay than the $\mathrm{g}_{2}$ at $850 \mathrm{~nm}$ due to the lower scattering and longer wavelength. By fixing $\mu_{s}{ }^{\prime}$ at $850 \mathrm{~nm}$ to $9.25 \mathrm{~cm}^{-1}$, we obtained an average $\mathrm{BF}_{\mathrm{i}}$ of $1.3 \pm 0.5 \times 10^{-8} \mathrm{~cm}^{2} / \mathrm{s}$ and a reduced scattering coefficient at $1064 \mathrm{~nm}$ of $8.39 \pm 1.40 \mathrm{~cm}^{-1}$, very close to the one reported in Table 2 . Since the measurements were not colocalized in each subject, some differences between the two wavelengths are expected. Figures 2(b) and 2(c) report the average CPS and $g_{2}(4 \mu \mathrm{s})$ SNR in each subject at the large SD separations for the two DCS systems. Figure 2(d) reports the theoretical and experimental $g_{2}(4 \mu \mathrm{s})$ SNRs, showing high correlations between theoretical and experimental SNR at all wavelengths and SD separations $(R 2=0.92$ for $850 \mathrm{~nm}$ at $25 \mathrm{~mm}, R 2=0.9$ for $1064 \mathrm{~nm}$ at $35 \mathrm{~mm}$, and $R 2=0.95$ for $1064 \mathrm{~nm}$ at $25 \mathrm{~mm}$ ). Finally, Figs. 2(e) and 2(f) show the CPS and $g_{2}(4 \mu \mathrm{s})$ SNR gain of $1064 \mathrm{~nm}$ with respect to $850 \mathrm{~nm}$ at a $25 \mathrm{~mm}$ SD separation. At the same separation, SNSPD-DCS collected an order of magnitude more photons than conventional DCS (average gain $13 \pm 6$ ) and for $\tau=4 \mu$ s we achieved an SNR gain of $16 \pm 8$. For $\tau$ ranging between 1 and $10 \mu$ s the average SNR gain was $18 \pm 10$. Moreover, with SNSPD-DCS, we were able to perform measurements at 35-mm separation while achieving a similar CPS and SNR as conventional DCS at $25 \mathrm{~mm}$. It is worth noting that in female subjects (\#2, 3, 6, 9, 10, and 11), we collect an average of $1.8 \pm 0.2$ times more photons and achieve $1.8 \pm 0.4$ times higher SNR than in male subjects, with the highest CPS and SNR gain at 1064 at $35 \mathrm{~mm}$.

The increased $g_{2}$ SNR allowed us to acquire data at $25 \mathrm{~mm}$ at faster rates and recover clean arterial pulsation signals at $20 \mathrm{~Hz}$ in all subjects (FFT pulsatile component CNR > 4). At $35 \mathrm{~mm}$
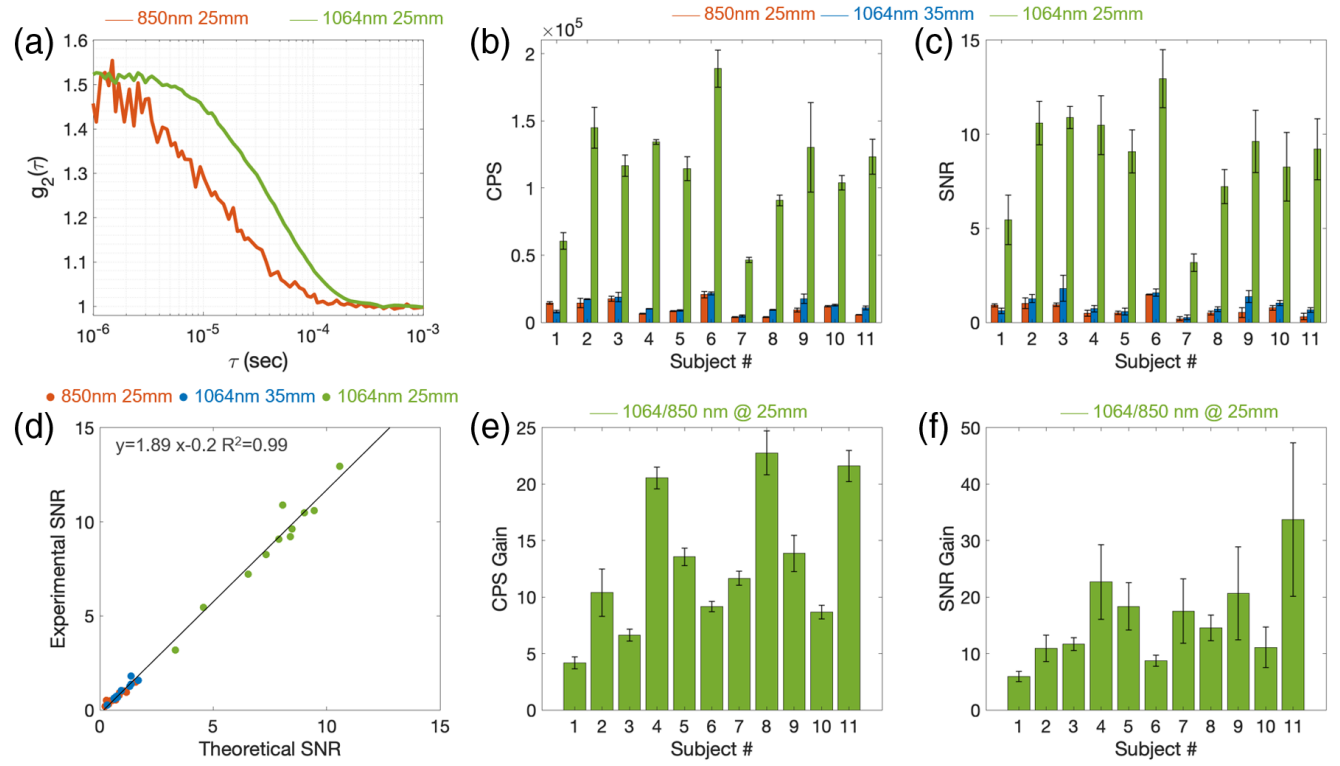

Fig. 2 (a) Autocorrelation curves acquired at $50 \mathrm{~Hz}$ and averaged over 50 diastolic points at 850 and $1064 \mathrm{~nm}$, both at a 25-mm SD separation, in a representative subject (\#6). The $g_{2}$ at $1064 \mathrm{~nm}$ is much less noisy and shows a slower decay, which further improves the SNR. (b) and (c) CPS and $g_{2}(4 \mu \mathrm{s})$ SNR in the 11 subjects at the three large separations. (d) Experimental versus theoretical $g_{2}(4 \mu \mathrm{s})$ SNR for all subjects. (e) and (f) CPS gain and $g_{2}(4 \mu \mathrm{s})$ SNR gain at $1064 \mathrm{~nm}$ $25 \mathrm{~mm}$ with respect to $850 \mathrm{~nm}$ at $25 \mathrm{~mm}$. In all bar-graphs each value represents the average and each errorbar represent the standard deviations across the three trials. In all panels, orange correspond to $850 \mathrm{~nm}$ at $25 \mathrm{~mm}$, blue correspond to $1064 \mathrm{~nm}$ at $35 \mathrm{~mm}$, and green correspond to $1064 \mathrm{~nm}$ at $25 \mathrm{~mm}$. 

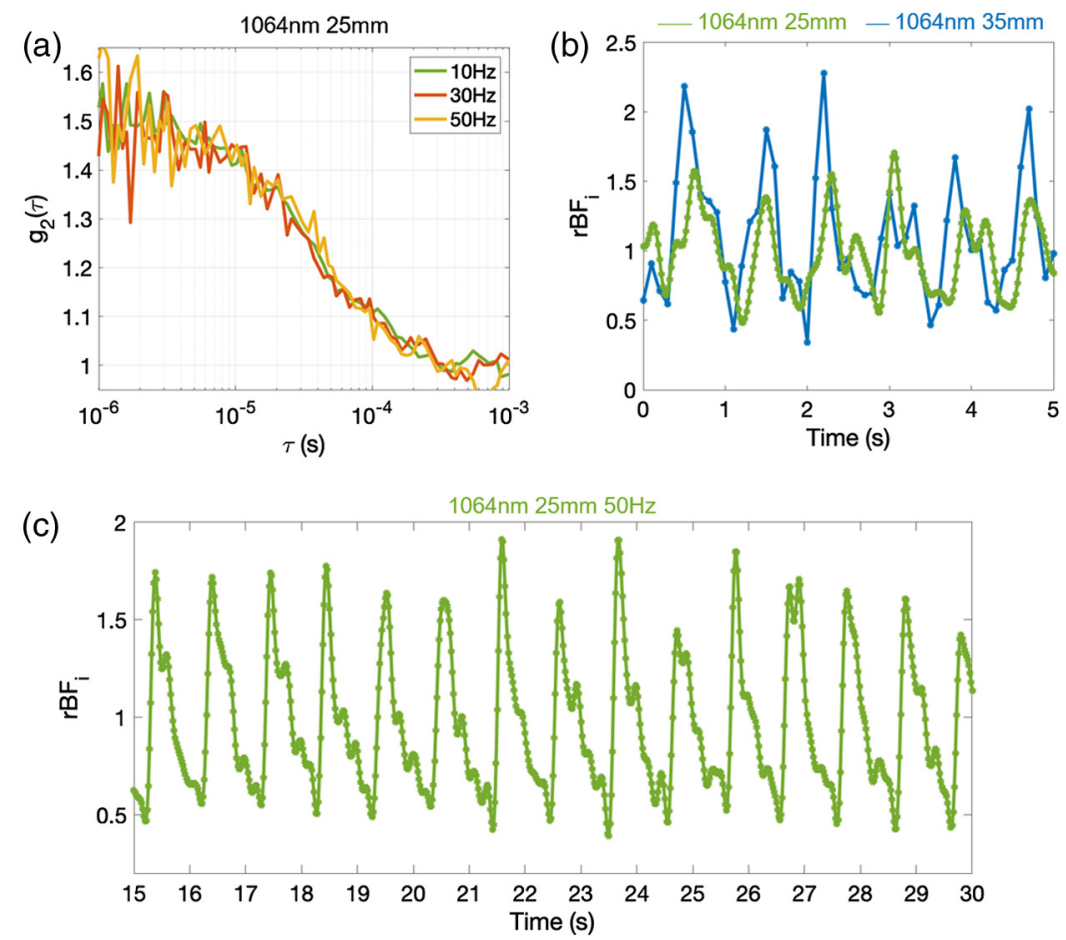

Fig. 3 (a) Example of an autocorrelation function acquired with the SNSPD-DCS at $25 \mathrm{~mm}$, estimated at three different frequencies, $10 \mathrm{~Hz}(100 \mathrm{~ms}$ integration time), $30 \mathrm{~Hz}(33 \mathrm{~ms})$, and $50 \mathrm{~Hz}$ $(20 \mathrm{~ms})$, on subject \#6. While the noise increases with frequency, the decay rate and the resulting $\mathrm{BF}_{\mathrm{i}}$ fit are consistent at all acquisition rates. (b) For the same subject as panel (a), we show $5 \mathrm{~s}$ of the $\mathrm{BF}_{\mathrm{i}}$ traces sampled at $50 \mathrm{~Hz}$ and $25 \mathrm{~mm}$ and at $10 \mathrm{~Hz}$ and $35 \mathrm{~mm}$ SD separations. (c) Example of $\mathrm{BF}_{\mathrm{i}}$ acquired at $50 \mathrm{~Hz}$ and $25 \mathrm{~mm}$ in subject \# $5(\mathrm{SNR}=9)$. A Butterworth bandpass filter of 0.1 to $5 \mathrm{~Hz}$ was applied to the data.

and $1064 \mathrm{~nm}$ and at $25 \mathrm{~mm}$ and $850 \mathrm{~nm}$, we achieved pulsatile CNR $>4$ in only four subjects (36\%) and only at $10 \mathrm{~Hz}$ acquisition rate. In these subjects, the CNR at $850 \mathrm{~nm}$ was $5.6 \pm 1.3$, lower than the CNR at $1064 \mathrm{~nm}(10 \pm 3.8)$ at the same SD separation, $25 \mathrm{~mm}$. Figure 3 shows examples of $g_{2}$ autocorrelation curves acquired at 10,30 , and $50 \mathrm{~Hz}$, and pulse waveforms at 25 and $35 \mathrm{~mm}$ with the SNSPD-DCS system.

\subsection{Sensitivity Estimates via Monte Carlo Simulation}

The Monte Carlo simulations on a 3D segmented head structure, as expected, showed increased sensitivity to the brain with increasing SD separations. As shown in Figs. 4(a) and 4(b), there is no substantial difference between cerebral blood flow (CBF) sensitivity at 850 and $1064 \mathrm{~nm}$ for the chosen optical properties (Table 2) when noise effects are not taken into account. Cerebral $\mathrm{BF}_{\mathrm{i}}$ sensitivity increases substantially at $35 \mathrm{~mm}$ with respect to the $25-\mathrm{mm}$ SD separation with an increase of $21.65 \%$ for a brain perturbation (20\% increase in $\mathrm{CBF}_{\mathrm{i}}$ ) and a decrease of $15 \%$ for a scalp $\mathrm{BF}_{\mathrm{i}}$ perturbation ( $85 \%$ scalp $\mathrm{BFi}$ reduction) at $1064 \mathrm{~nm}$. This is in agreement with our experimental results where for averaged data (acquisition rate $0.1 \mathrm{~Hz}$ ) we did not observe significant differences in $\mathrm{BF}_{\mathrm{i}}$ changes at $25 \mathrm{~mm}$ at the two wavelengths, but we observed significant improvements at $35-\mathrm{mm}$ SD separation, only achievable at $1064 \mathrm{~nm}$ (SNR at $850 \mathrm{~nm}$ is too low at this separation even when acquiring at $0.1 \mathrm{~Hz}$ or less). We then add noise to the simulations based on the CPS obtained experimentally at 25- and 35-mm SD separations, and compute the expected contrast-to-noise-ratio of CBF measurement. As shown in Fig. 4(c) (CNR normalized by the maximum value) the improvement in the performance of the SNSPD-DCS at $1064 \mathrm{~nm}$ with respect to the Si-SPAD-based DCS at $850 \mathrm{~nm}$ are clear. As separation increases, noise rises much faster than intrinsic sensitivity, leading to an optimal separation range for each wavelength. This optimal SD separation at $850 \mathrm{~nm}$ is $25 \mathrm{~mm}$, whereas for the SNSPD-DCS at $1064 \mathrm{~nm}$, 
(a)

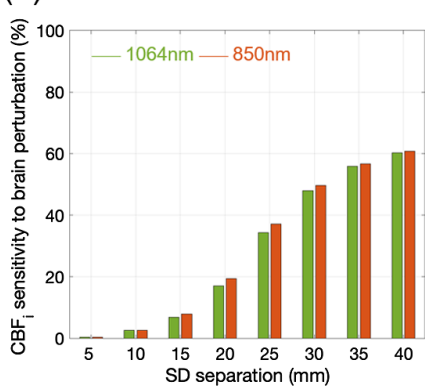

(b)

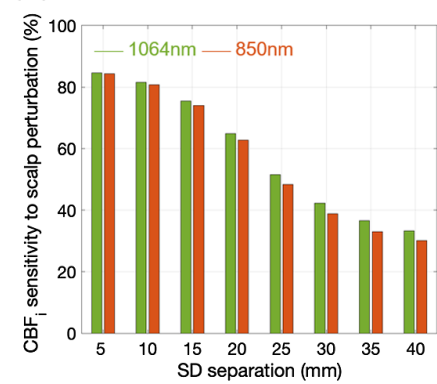

(c)

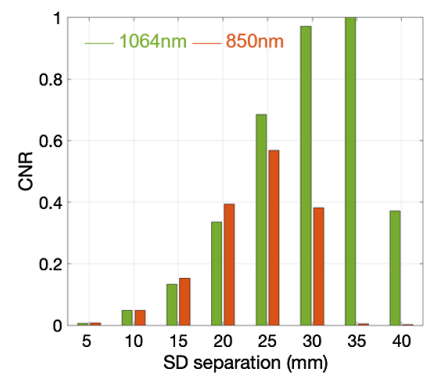

Fig. 4 CBF index sensitivity as a function of SD separation at 850 and $1064 \mathrm{~nm}$ for (a) a brain perturbation (20\% increase in $\mathrm{CBFi}$ ) and (b) a scalp perturbation (85\% increase in scalp $\mathrm{BFi}$ ). (c) Brain contrast-to-noise ratio (normalized by the maximum value) as a function of SD separation at 850 and $1064 \mathrm{~nm}$ at $1 \mathrm{~Hz}$ acquisition rate.

the optimal SD separation is $35 \mathrm{~mm}$, which allows for a $31.6 \%$ relative increase in brain blood flow sensitivity.

\subsection{Pressure Modulation Results}

Subjects \#1 and \#6 and trials 2.1, 4.1, 4.2, 7.1, 8.1, 9.1, and 9.2 were excluded based on the criteria defined in Sec. 2.5 (a $>10 \%$ difference in the reduction of the two short separation wavelengths). No statistical difference was found on the remaining trials between the short separations at 1064 and $850 \mathrm{~nm}$, with an average reduction at the $5 \mathrm{~mm}$ SD separations of $89.4 \pm 4.5$ at $850 \mathrm{~nm}$ and of $93.4 \pm 3.7$ at $1064 \mathrm{~nm}(p=0.08)$. Figure 5(a) shows an example of $\mathrm{rBF}_{i}$ versus time during a trial (subject \#5 trial \#3). Figure 5(b) and 5(c) report the average reductions for each trial and the grand averages across all trials. While we did not find statistically significant differences between the reductions at $25 \mathrm{~mm}$ for either wavelength (48.1 \pm 7.7 and $48.0 \pm 8.4$, $p=0.99$ ), we consistently observed a lower reduction for the $35 \mathrm{~mm}$ at $1064 \mathrm{~nm}$ measurements, equal to $30.7 \pm 6.4$, with $p=3.6 \times 10^{-4}$ for the comparison with $25 \mathrm{~mm}$ at $850 \mathrm{~nm}$, and $p=1.52 \times 10^{-4}$ for the comparison with $25 \mathrm{~mm}$ at $1064 \mathrm{~nm}$. The experimental result is in good agreement with the Monte Carlo simulations [Fig. 4(b)]. At 25-mm SD separation, the experimental $\mathrm{BF}_{\mathrm{i}}$ reduction of $48 \%$ and $48.1 \%$, at 850 and $1064 \mathrm{~nm}$, respectively, match the simulation reductions ( $48.1 \%$ and $51.5 \%$, at 850 and $1064 \mathrm{~nm}$, respectively). At $35 \mathrm{~mm}$ and $1064 \mathrm{~nm}$, the experimental reduction of $30.7 \%$ is also similar to the simulation $\mathrm{BF}_{\mathrm{i}}$ reduction $(36.5 \%)$.

As shown in Fig. 5, the main advantage of SNSPD-DCS is that we are able to perform measurements at larger SD separations and increase the sensitivity to cerebral changes in all subjects.

In addition to a reduction in $\mathrm{BF}_{\mathrm{i}}$, the compression also drastically reduced the pulsatile waveform at the short separations. Conversely, the amplitude of the pulsatile blood flow at the larger separations remains substantial, with an average reduction of $35.5 \pm 29 \%$ at 25 and $1064 \mathrm{~nm}$. An example is reported in Fig. 6. This suggests that a large component of the pulsatile $\mathrm{BF}_{\mathrm{i}}$ signal originates below the scalp and skull.

\subsection{Breathing Tasks Results}

These experiments were conducted to induce and measure differences in cerebral versus peripheral responses to systemic perturbations. Subjects 4 and 8 (except 8.3 breath-holding) were excluded because of large oscillations of the $\mathrm{BF}_{\mathrm{i}}$ at short separations. Subject 9 did not perform the first hyperventilation trial. For breath-holding, we divided the trials in two groups. In the first group, we measured an $\mathrm{SpO}_{2}$ decrease in response to breath-holding of $\geq 5 \%(1.1,1.2,1.3,2.2$, $3.1,5.2,6.1,6.2,6.3,8.3,11.1,11.2,11.3 ; 13$ trials). The second group had trials for whom $\mathrm{SpO}_{2}$ responses <5\% (2.1, 2.3, 3.2, 3.3, 5.1, 5.3, 7.1, 7.2, 7.3, 9.1, 9.2, 9.3, 10.1, 10.2, 10.3; 15 trials). For the hyperventilation results, we excluded trials 2.3 and 9.3 because the HR changed $<10 \mathrm{bpm}$ as a consequence of the fast breathing, leaving us with 24 trials. 

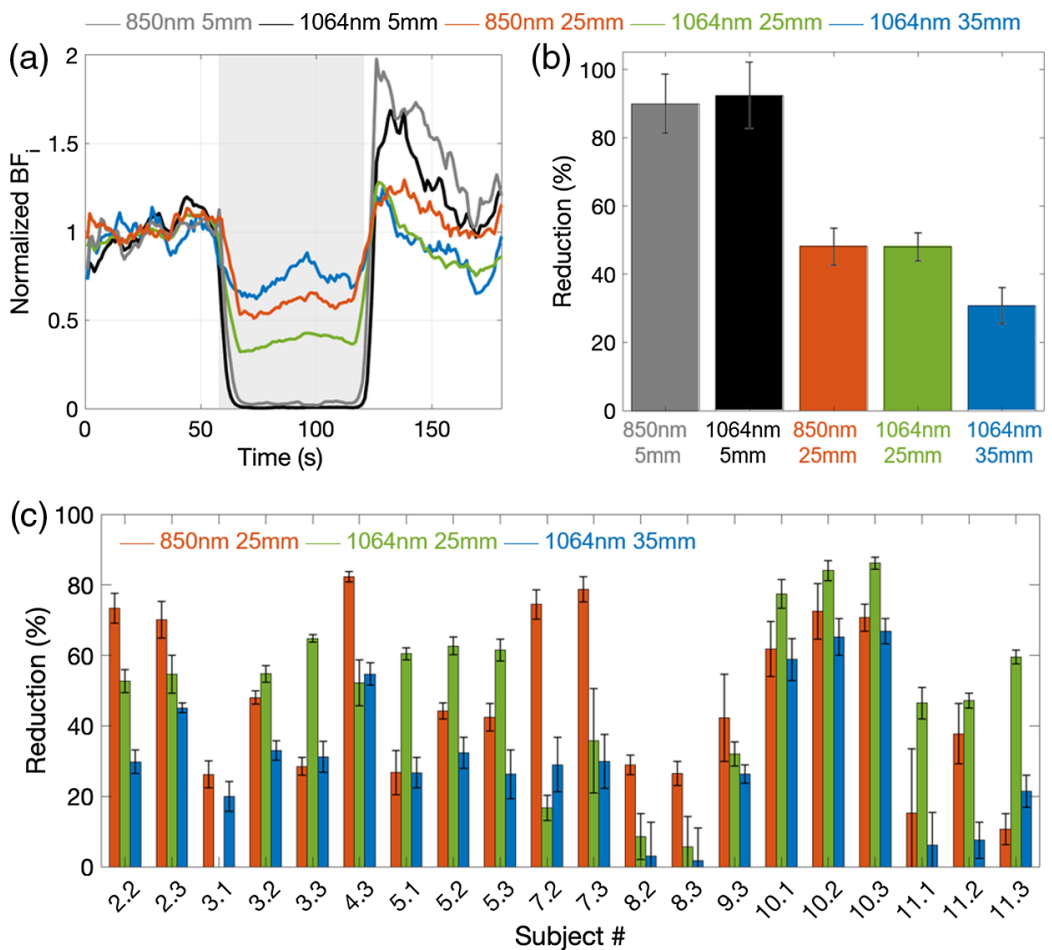

Fig. 5 (a) An example of a pressure modulation trial (subject \#5, trial \#3), reporting $\mathrm{rBF}_{\mathrm{i}}$ at $1 \mathrm{~Hz}$ for all SD separations of the two DCS systems. The gray shaded area represents the period during which the tourniquet was tightened. (b) Percent reduction in $\mathrm{rBF}_{\mathrm{i}}$ during pressure with respect to initial baseline at all SD separations averaged across all trials and subjects. Error bars represent standard errors across all subjects. (c) Percent reduction in $\mathrm{rBF}_{\mathrm{i}}$ during pressure with respect to initial baseline at the large SD separations for each included trial. Error bars represent standard deviations during the compression periods.

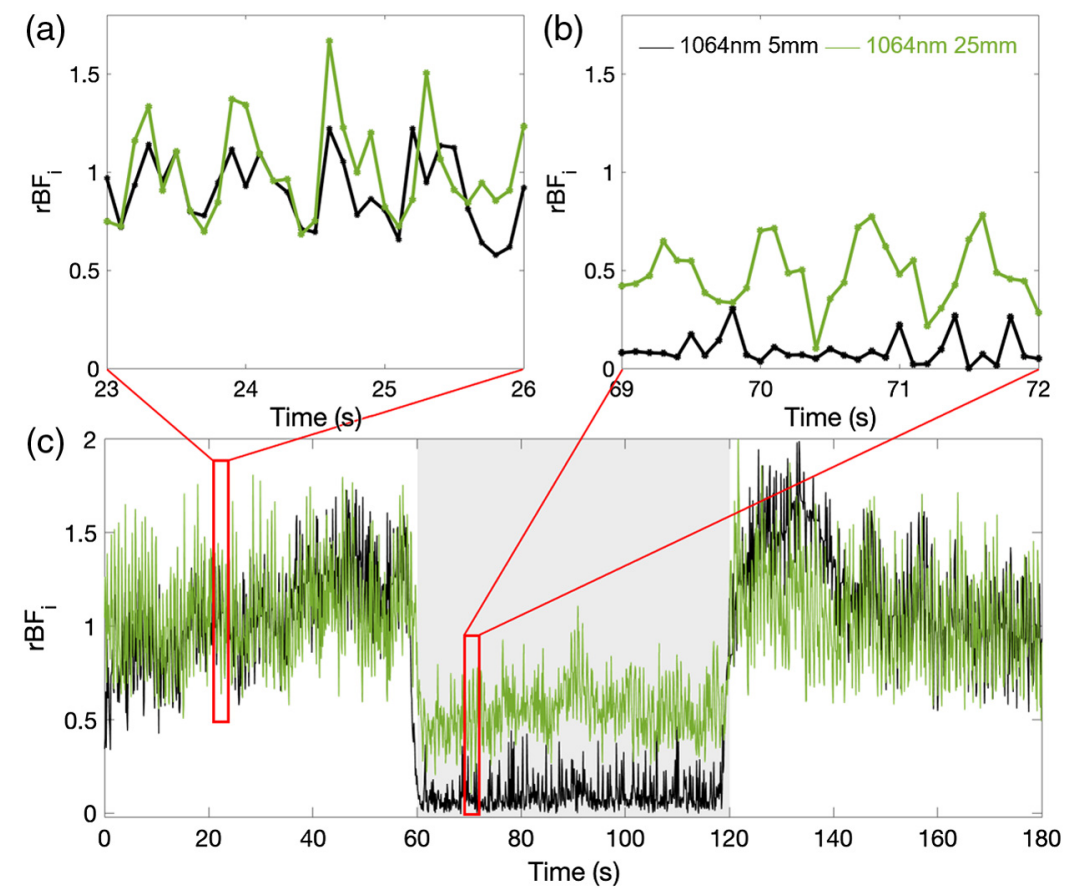

Fig. $6 \mathrm{rBF}_{\mathrm{i}}$ during a pressure modulation trial on a representative subject (\#11.1) acquired at $10 \mathrm{~Hz}$. Results at 5- and 25-mm SD separations at $1064 \mathrm{~nm}$ are reported in figures. The large fluctuation on the signal is not noise but the pulsatile blood flow oscillations. Panels (a) and (b) give an expanded view of two 3-s periods during baseline and pressure. 

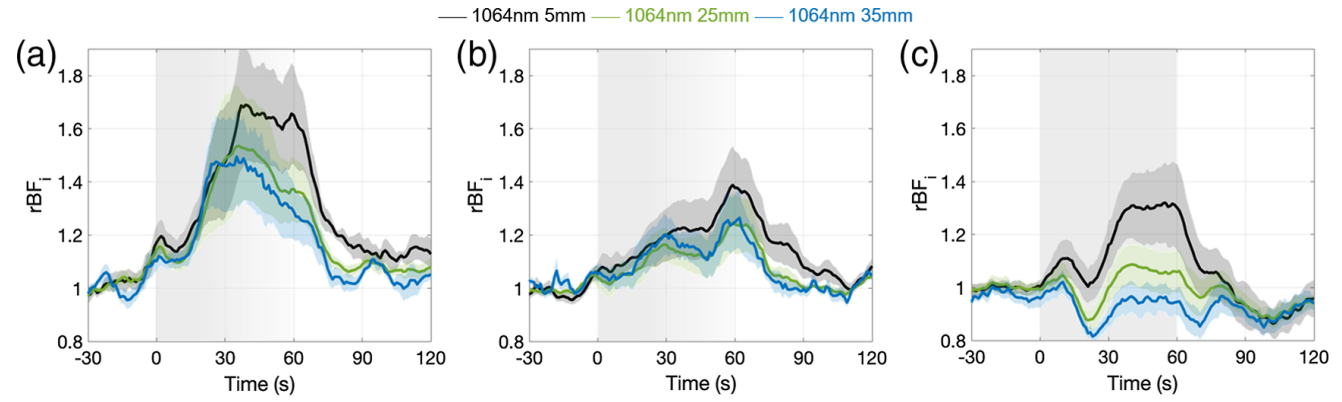

Fig. 7 Breathing tasks $\mathrm{BF}_{\mathrm{i}}$ results. (a) $\mathrm{BF}_{\mathrm{i}}$ changes during breath-holding task averaged across group 1 trials $\left(\mathrm{SpO}_{2}\right.$ reduction $\left.\geq 5 \%\right)$. (b) $\mathrm{BF}_{\mathrm{i}}$ changes during breath holding task for group $2\left(\mathrm{SpO}_{2}\right.$ reduction $<5 \%$ ). (c) $\mathrm{BF}_{\mathrm{i}}$ changes during hyperventilation average across all included trials. Time zero correspond to the start of breath-holding or hyperventilation. While all subjects performed a breath-holding of at least $25 \mathrm{~s}$, only few were able to maintain it for $60 \mathrm{~s}$ (shown as a gray gradient shaded period in the figures). For hyperventilation, all subjects performed it for $60 \mathrm{~s}$. Shaded areas represent standard errors across subject.

Figure 7 reports the relative $\mathrm{BF}_{\mathrm{i}}$ changes for the different groups and tasks measured with the SNSPD-DCS system at $1064 \mathrm{~nm}$, with Figs. 7(a) and 7(b) reporting the $\mathrm{rBF}_{\mathrm{i}}$ responses to the breath-holding task in groups $1\left(\mathrm{SpO}_{2}\right.$ drop $\left.\geq 5 \%\right)$ and $2\left(\mathrm{SpO}_{2}\right.$ drop $\left.<5 \%\right)$ and Fig. 7 (c) reporting $\mathrm{rBF}_{\mathrm{i}}$ changes with hyperventilation. As expected, blood flow increases during the first $30 \mathrm{~s}$ in response to breath-holding due to transient hypercapnic hypoxia and slowly returns to baseline during the second phase of breath-holding due to a high concentration of $\mathrm{CO}_{2}$ in the blood stream and a deficiency of $\mathrm{O}_{2}$ in the tissues. Larger changes were observed in the group with larger $\mathrm{SpO}_{2}$ drops $(69 \pm 19.3 \%$ in group 1 versus $38 \pm 9.6 \%$ in group 2$)$. In both panels a and $b$, we can see faster responses at larger separations, due to the faster reactivity of the brain than the scalp. ${ }^{25}$ Statistically significant differences between $\mathrm{rBF}_{\mathrm{i}}$ at 35 and $5 \mathrm{~mm}$ SD separations were found at times 63 to $88 \mathrm{~s}$ and 108 to $118 \mathrm{~s}(p<0.05)$, Statistically significant differences between $\mathrm{rBF}_{\mathrm{i}}$ at 25 and $5 \mathrm{~mm}$ were found at times 63 to $84 \mathrm{~s}$ and 108 to $109 \mathrm{~s}$ $(p<0.05)$. No statistically significant differences were found between responses at 35 and $25 \mathrm{~mm}$.

The hyperventilation task causes hypocapnia, and while the scalp blood flow increases due to the large increase in HR during fast paced breathing, the $\mathrm{CBF}$ decreases due to vasoconstriction. As shown in Fig. 7(c), we clearly observed this differential behavior between scalp and brain in the $\mathrm{rBF}_{\mathrm{i}}$ responses at 5 and $35 \mathrm{~mm}$, with responses at $25 \mathrm{~mm}$ in between the two, because of the lower sensitivity to brain at this SD separation. Specifically, $\mathrm{rBF}_{\mathrm{i}}$ at $5 \mathrm{~mm}$ showed an increase of $31.9 \pm 13.8 \%$ and $\mathrm{rBFi}$ at $35 \mathrm{~mm}$ showed a decrease of $18.5 \pm 2.3 \%$. Statistically significant differences between $\mathrm{rBF}_{\mathrm{i}}$ at 35 and $5 \mathrm{~mm} \mathrm{SD}$ separations were found at times 0 to $78 \mathrm{~s}$ $(\mathrm{p}<0.05)$. Statistically significant differences between $\mathrm{rBF}_{\mathrm{i}}$ at 25 and $5 \mathrm{~mm}$ between 0 and $72 \mathrm{~s}$. Statically significant differences between $\mathrm{rBF}_{\mathrm{i}}$ at $25-$ and $35 \mathrm{~mm}-\mathrm{SD}$ separations were found at times 24 to $85 \mathrm{~s}(p<0.05)$.

Breath-holding and hyperventilation responses at $850 \mathrm{~nm}$ had similar trends, with within and across subject's differences due to the different measurement locations at the two wavelengths (not shown). We quantified the $\mathrm{rBF}_{\mathrm{i}}$ changes at $850 \mathrm{~nm}$ and $1064 \mathrm{~nm}$ (at the same SD separation, $25 \mathrm{~mm}$ ) during the breathing tasks via Bland-Altman analysis. For the maximum $\mathrm{rBF}_{\mathrm{i}}$ changes in the breath-holding groups I and II and in the hyper-ventilation (24 trials), 94.23\% (49 out of 52) of the data points (i.e., relative changes at 1064 and $850 \mathrm{~nm}$ ) fall within $\pm 1.96 \mathrm{STD}$ of the mean difference, due to the similar trends between the two wavelengths.

\section{Discussion and Conclusions}

This is the first report demonstrating a high signal-to-noise ratio with homodyne DCS at an SD separation of $35 \mathrm{~mm}$ in-vivo on a substantial number of subjects. As described in Sec. 2, the advantages of the presented method were achieved by using a single channel SNSPD and 
$1064 \mathrm{~nm}$ illumination. The major contribution to the SNR improvement with respect to conventional DCS is given by the 7 to 8 times more photons available at the detector at 1064 than at $850 \mathrm{~nm}$, as reported in Ref. 10. An additional contribution to the total photon gain at 1064 with respect to $850 \mathrm{~nm}$ is given by the 1.5 times higher photon detection efficiency (PDE) of the SNSPD used here $(\sim 88 \%$ at $1064 \mathrm{~nm})$ and the extremely low dark count rate $(1 \mathrm{CPS})$ with respect to the Si-SPAD PDE ( $58 \%$ at $850 \mathrm{~nm})$ and dark count rate (1500 CPS). Together, these two factors provide an overall experimental averaged gain of 13 in photon count at 25-mm SD separation and, by also considering the slower decay of the autocorrelation function at the longer wavelength, an averaged $\mathrm{g}_{2}(4 \mu \mathrm{s})$ SNR gain of 16 . It is worth noting that $\mathrm{g}_{2}$ SNR increases in direct proportionality to the instantaneous count rate, whereas it only increases with the square root of the acquisition time or multi-channel averaging. Thus, for standard DCS, to achieve the same $\mathrm{g}_{2}$ SNR gain achieved here one would need to increase the integration times or the number of channels by a factor of $256(\sim 16=\sqrt{256})$.

By using SNSPD also at $850 \mathrm{~nm}$ the photon budget improvement at $1064 \mathrm{~nm}$ versus $850 \mathrm{~nm}$ would have been 1.5 time smaller than what measured using Si-SPADs at 850 and SNSPD at $1064 \mathrm{~nm}$. Using Si-SPAD at $1064 \mathrm{~nm}$ would have drastically reduced the 1064 photon budget since the PDE of the Si-SPAD at 1064 is $<3 \%$.

The increased SNR of the SNSPD-DCS at $1064 \mathrm{~nm}$ allowed us to resolve clear arterial pulsation at $20 \mathrm{~Hz}$ at a $25 \mathrm{~mm}$ SD separation in all subjects. Moreover, as previously observed by Wang et al. ${ }^{26}$ we found the pulsatile blood flow component at this SD separation is less affected by scalp interference than the average blood flow signal as shown during the pressure modulation experiment. In fact, during compression at 25 and $1064 \mathrm{~nm}$ we observed a $\mathrm{BF}_{\mathrm{i}}$ reduction of $48.1 \%$ and an attenuation of pulsatile flow of $35 \%$. Being able to acquire pulsatile blood flow at high frequency with low SNR at separations of $25 \mathrm{~mm}$ or more, is important in applications where pulsatile blood flow is used to estimate intracranial pressure non-invasively. ${ }^{17,27}$

In agreement with the Monte Carlo simulations, we observed improved brain sensitivity in our experiments by increasing SD separation. The pressure modulation results show a smaller reduction in $\mathrm{rBF}_{\mathrm{i}}$ at $35 \mathrm{~mm}$ separation with respect to $25 \mathrm{~mm}$ (both 850 and $1064 \mathrm{~nm}$ ) and the breathing tasks shows more consistent statistically significant differences in $\mathrm{rBF}_{\mathrm{i}}$ between 5 and $35 \mathrm{~mm} . \mathrm{rBF}_{\mathrm{i}}$ increases of $69 \%$ during breath-holding and decreases of $18.5 \%$ during hyperventilation are in agreement with MRI and PET studies. ${ }^{25,28,29}$

While in some subjects we obtain similar responses at 25 and $35 \mathrm{~mm}$, the use of $35 \mathrm{~mm}$ allows recovery of CBF changes more consistently across subjects. This is needed to provide better accuracy and consistent efficacy when moving to adult clinical applications.

\section{Disclosures}

MAF has a financial interest in 149 Medical, Inc., a company developing DCS technology for assessing and monitoring CBF in newborn infants. MAF's interests were reviewed and are managed by Massachusetts General Hospital and Partners HealthCare in accordance with their conflicts of interest policies.

\section{Acknowledgments}

This work was supported by the National Institutes of Health (NIH), Award No. U01EB028660. The authors would like to thank Tim Rambo and Aaron Miller from Quantum Opus and Nick Bertone from OptoElectronic Components. The authors would like to thank Zachary Starkweather for the optical probes fabrication, Marco Renna for help with the hardware, John Sunwoo for discussions on the statistical analysis, and Kuan Chang (Tony) Wu for help with signal processing.

\section{Code, Data, and Materials Availability}

Data supporting the results reported in the manuscript may be requested by contacting the corresponding author. 


\section{References}

1. E. M. Buckley et al., "Diffuse correlation spectroscopy for measurement of cerebral blood flow: future prospects," Neurophotonics 1(1), 011009 (2014).

2. D. A. Boas et al., "Establishing the diffuse correlation spectroscopy signal relationship with blood flow," Neurophotonics 3(3), 031412 (2016).

3. T. Durduran and A. G. Yodh, "Diffuse correlation spectroscopy for non-invasive, microvascular cerebral blood flow measurement," NeuroImage 85, 51-63 (2014).

4. G. Dietsche et al., "Fiber-based multispeckle detection for time-resolved diffusing-wave spectroscopy: characterization and application to blood flow detection in deep tissue," Appl. Opt. 46(35), 8506-8514 (2007).

5. J. D. Johansson et al., "A multipixel diffuse correlation spectroscopy system based on a single photon avalanche diode array," J. Biophotonics 12(11), e201900091 (2019).

6. E. J. Sie et al., "High-sensitivity multispeckle diffuse correlation spectroscopy," Neurophotonics 7(3), 035010 (2020).

7. M. B. Robinson et al., "Interferometric diffuse correlation spectroscopy improves measurements at long source-detector separation and low photon count rate," J. Biomed. Opt. 25(9), 097004 (2020).

8. W. Zhou et al., "Highly parallel, interferometric diffusing wave spectroscopy for monitoring cerebral blood flow dynamics," Optica 5(5), 518-527 (2018).

9. E. James and S. Powell, "Fourier domain diffuse correlation spectroscopy with heterodyne holographic detection," Biomed. Opt. Express 11(11), 6755-6779 (2020).

10. S. A. Carp et al., "Diffuse correlation spectroscopy measurements of blood flow using 1064 nm light," J. Biomed. Opt. 25(9), 097003 (2020).

11. C. M. Natarajan, M. G. Tanner, and R. H. Hadfield, "Superconducting nanowire singlephoton detectors: Physics and applications," Supercond. Sci. Technol. 25(6), 063001 (2012).

12. G. N. Gol'tsman et al., "Picosecond superconducting single-photon optical detector," Appl. Phys. Lett. 79(6), 705-707 (2001).

13. H. Wang et al., "Large-area multispectral superconducting nanowire single-photon detector," Appl. Opt. 58(30), 8148-8152 (2019).

14. H. Shibata et al., "Ultimate low system dark-count rate for superconducting nanowire single-photon detector," Opt. Lett. 40(14), 3428-3431 (2015).

15. R. H. Hadfield, "Single-photon detectors for optical quantum information applications," Nat. Photonics 3(12), 696-705 (2009).

16. L. M. Hirvonen and K. Suhling, "Fast timing techniques in FLIM applications," Front. Phys. 8, 161 (2020).

17. W. B. Baker et al., "Probe pressure modulation algorithm reduces extracerebral contamination in optical measurements of cerebral blood flow," in Biomed. Opt., OSA, paper BS3A.52 (2014).

18. J. S. Meyer et al., "Cerebral hemodynamics, blood gases, and electrolytes during breathholding and the Valsalva maneuver," Circulation 33(5 Suppl.), 33:II-35-II-48 (1966).

19. C. Cheung et al., "In vivo cerebrovascular measurement combining diffuse near-infrared absorption and correlation spectroscopies," Phys. Med. Biol. 46(8), 2053-2065 (2001).

20. A. Farina et al., "In-vivo multilaboratory investigation of the optical properties of the human head," Biomed. Opt. Express 6(7), 2609-2623 (2015).

21. D. E. Koppel, "Statistical accuracy in fluorescence correlation spectroscopy," Phys. Rev. A 10(6), 1938-1945 (1974).

22. C. Zhou, "In-vivo optical imaging and spectroscopy of cerebral hemodynamics," Ph.D. Dissertation, University of Pennsylvania (2007).

23. Q. Fang and D. A. Boas, "Monte Carlo simulation of photon migration in 3D turbid media accelerated by graphics processing units," Opt. Express 17(22), 20178 (2009).

24. D. Mazumder et al., "Optimization of time domain diffuse correlation spectroscopy parameters for measuring brain blood flow," Neurophotonics 8(3), 035005 (2021).

25. A. Kastrup et al., "Cerebral blood flow-related signal changes during breath-holding," Am. J. Neuroradiol. 20(7), 1233-1238 (1999). 
26. D. Wang et al., "Influence of probe pressure on the pulsatile diffuse correlation spectroscopy blood flow signal on the forearm and forehead regions," Neurophotonics 6(3), 035013 (2019).

27. K.-C. Wu et al., "Validation of diffuse correlation spectroscopy measures of critical closing pressure against transcranial Doppler ultrasound in stroke patients," J. Biomed. Opt. 26(3), 036008 (2021).

28. A. W. Pan et al., "Blood flow in the carotid artery during breath-holding in relation to diving bradycardia," Eur. J. Appl. Physiol. Occupational Physiol. 75(5), 388-395 (1997).

29. R. F. Albrecht, D. J. Miletich, and M. Ruttle, "Cerebral effects of extended hyperventilation in unanesthetized goats," Stroke 18(3), 649-655 (1987).

30. A. Torricelli et al., "In vivo optical characterization of human tissues from 610 to $1010 \mathrm{~nm}$ by time-resolved reflectance spectroscopy," Phys. Med. Biol. 46(8), 2227 (2001).

31. M. Firbank et al., "Measurement of the optical properties of the skull in the wavelength range 650-950 nm," Phys. Med. Biol. 38(4), 503-510 (1993).

Biographies of the authors are not available. 